\title{
Estudo da dinâmica espaço-temporal do bioma Pantanal por meio de imagens MODIS
}

\author{
Marcos Adami( ${ }^{(1)}$, Ramon Morais de Freitas ${ }^{(1)}$, Carlos Roberto Padovani(2), Yosio Edemir Shimabukuro ${ }^{(1)}$ \\ e Mauricio Alves Moreira(1)
}

(1)Instituto Nacional de Pesquisas Espaciais, Caixa Postal 515, CEP 12245-970 São José dos Campos, SP. E-mail: adami@dsr.inpe.br, ramon@dsr.inpe.br, yosio@dsr.inpe.br, mauricio@dsr.inpe.br ${ }^{(2)}$ Embrapa Pantanal, Caixa Postal 109, CEP 79320-900 Corumbá, MS. E-mail: guara@cpap.embrapa.br

\begin{abstract}
Resumo - O objetivo deste trabalho foi avaliar dados multitemporais, obtidos pelo sensor "moderate resolution imaging spectroradiometer" (MODIS), para o estudo da dinâmica espaço-temporal de duas sub-regiões do bioma Pantanal. Foram utilizadas 139 imagens "enhanced vegetation index" (EVI), do produto MOD13 "vegetation index", dados de altimetria oriundos do "shuttle radar topography mission" (SRTM) e dados de precipitação do "tropical rainfall measuring mission" (TRMM). Para a redução da dimensionalidade dos dados, as imagens MODIS-EVI foram amostradas com base nas curvas de nível espaçadas em $10 \mathrm{~m}$. Foram aplicadas as técnicas de análise de autocorrelação e análise de agrupamentos aos dados das amostras, e a análise de componentes principais na área total da imagem. Houve dependência tanto temporal quanto espacial da resposta espectral com a precipitação. A análise de agrupamentos apontou a presença de dois grupos, o que indicou a necessidade da análise completa da área. A análise de componentes principais permitiu diferenciar quatro comportamentos distintos: as áreas permanentemente alagadas; as áreas não inundáveis, compostas por vegetação; as áreas inundáveis com maior resposta de vegetação; e áreas com vegetação ripária.
\end{abstract}

Termos para indexação: sensoriamento remoto, série temporal, análise de agrupamento.

\section{Spatial-temporal analysis of MODIS image applied to dynamic of Pantanal biome}

\begin{abstract}
The objective of this work was to evaluate multitemporal data, obtained by moderate resolution imaging spectroradiometer (MODIS) sensor, for the study of spatial-temporal dynamics in two subregions of the Pantanal biome. One hundred and thirty nine enhanced vegetation index (EVI) images, from MOD13 vegetation index product, altimetry data from shuttle radar topography mission (SRTM) and tropical rainfall measuring mission (TRMM) precipitation data were used. In order to reduce data dimensionality, MODIS EVI images were sampled based on contour lines spacing of $10 \mathrm{~m}$. The autocorrelation and cluster analysis were used for spatial and temporal evaluation of the samples; and the principal components analysis was applied to all dataset for spatial and temporal analysis. Results showed a spatial and temporal dependence between spectral response and precipitation. The cluster analysis indicated two spatial groups, suggesting the need for the analysis of the entire study area. The principal components analysis allowed to distinguish four behaviors: the areas permanently flooded; nonflooded areas composed by vegetation; flooded areas with higher spectral vegetation response; and riparian vegetation areas.
\end{abstract}

Index terms: remote sensing, time series, cluster analysis.

\section{Introdução}

Segundo Adamoli (1995), o regime de inundações é o fator ecológico fundamental do Pantanal que determina os pulsos dos principais processos bióticos e abióticos, bem como as composições específicas das unidades de paisagem. Alterações no regime de inundações causam mudanças na cobertura vegetal, o que influencia todo o bioma. Porém, o monitoramento dessas áreas é uma tarefa difícil, em razão das grandes extensões de terra e da dificuldade de acesso (Pott \& Pott, 2004).

De acordo com Shimabukuro et al. (1998), alterações na umidade do solo, no balanço hídrico e na cobertura vegetal podem ser detectadas pela análise de dados de sensoriamento remoto. Entretanto, estudos multitemporais ficam limitados a sensores com menor resolução espacial como, por exemplo, o “advanced very high resolution radiometer" (AVHRR). Porém, a 
qualidade geométrica, a resolução espacial e a espectral deste sensor dificultam estudos em escala local.

Lançado em dezembro de 1999, com início de operação em fevereiro de 2000, o sensor "moderate resolution imaging spectroradiometer" (MODIS) tem como objetivo geral fornecer suporte a três segmentos do projeto "Earth Observing System" (EOS): atmosfera, oceano e continente. Esse sensor permitiu avanços nos trabalhos que necessitam de maior resolução temporal (quase diária), aliada à moderada resolução espacial $(250 \mathrm{~m})$. A qualidade do georreferenciamento das imagens MODIS permite a elaboração de séries temporais com precisão (Wolfe et al., 2002). Ao se combinarem as características das imagens (qualidade geométrica, radiométrica, espectral e temporal) com os algoritmos de geração de produtos validados (Justice et al., 2002), é possível estimar, por exemplo, os parâmetros biofísicos da vegetação, entre outros. Assim, os produtos voltados para aplicações terrestres, gerados pelo MODIS, estão basicamente direcionados para o monitoramento da cobertura vegetal do planeta, para identificar mudanças decorrentes de variações no clima e tempo (Justice et al., 2002).
O objetivo deste trabalho foi avaliar a dinâmica espaço-temporal, por meio de imagens MODIS, em duas sub-regiões do bioma Pantanal.

\section{Material e Métodos}

$\mathrm{O}$ estudo foi realizado nas sub-regiões sul-matogrossenses do Paiaguás, com $27.082 \mathrm{~km}^{2}$, e da Nhecolândia, com $26.921 \mathrm{~km}^{2}$ (Silva \& Abdon, 1998) (Figura 1).

A vegetação da região é um mosaico fragmentado, dominado por cerrado, com formações que variam de seus tipos arbóreo-densa a gramíneo-lenhosa, podendose, também, encontrar feições de estepe sujeitas à inundação (campos inundáveis) e floresta estacional em locais com pouca ou nenhuma inundação, tais como cordilheiras, capões e borda da planície (IBGE, 1992; Abdon et al., 1998; Pott \& Pott, 2004). Em sua maioria são terrenos quaternários aluviais, com altitudes abaixo de $200 \mathrm{~m}$ (Pott \& Pott, 2004). O solo é altamente arenoso, com predominância do Espodossolo hidromórfico e do Neossolo Quartzarênico hidromórfico (Embrapa, 2006). O clima é tropical de Savana (AW), conforme
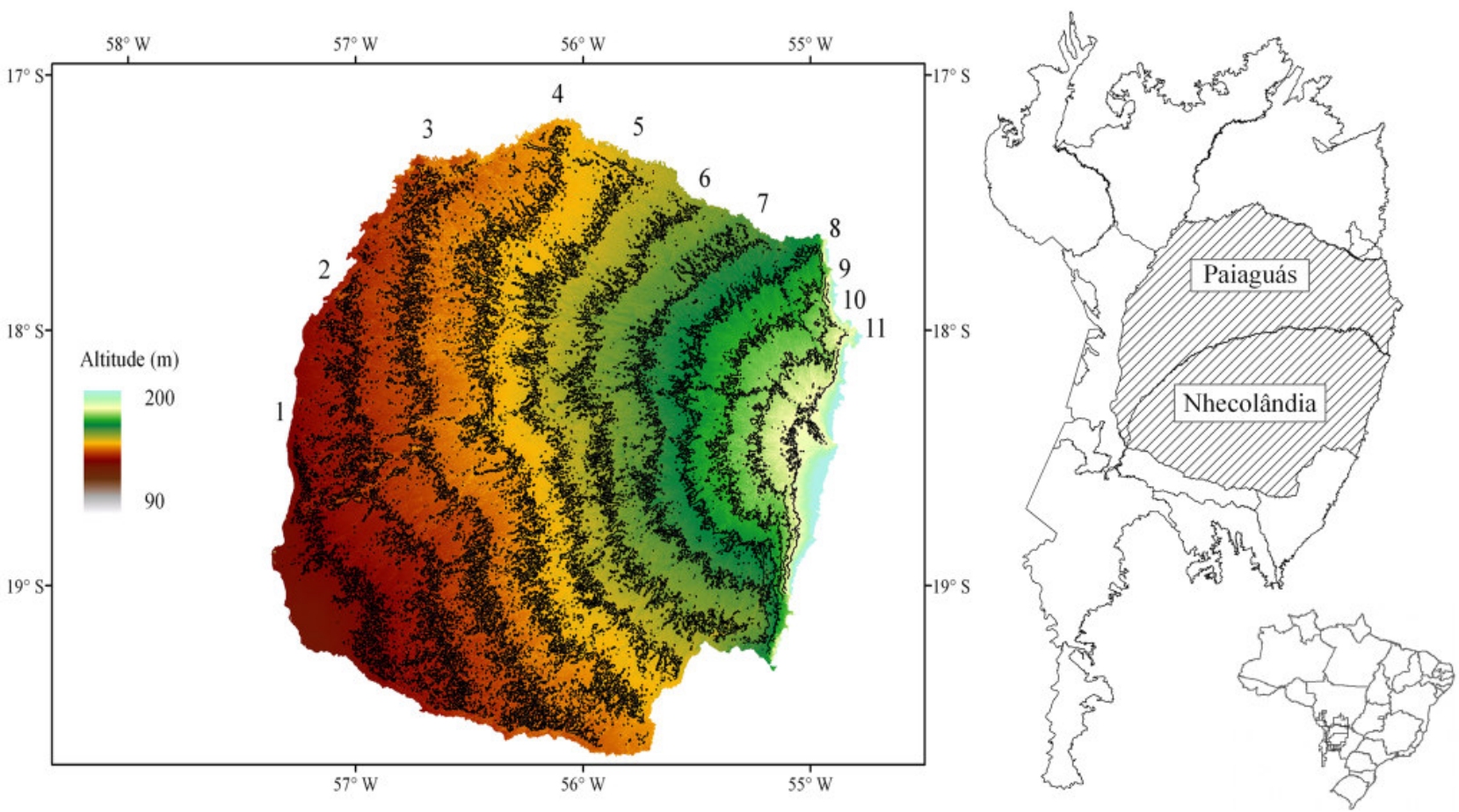

Figura 1. Localização da área de estudo no bioma Pantanal, com ênfase aos dados altimétricos do "shuttle radar topography mission", inclusive cotas amostradas, utilizadas neste trabalho, que variam de 90 a $190 \mathrm{~m}$, numeradas em ordem crescente de altitude. 
Köppen, que é caracterizado por períodos anuais de chuva e estiagem, coincidentes ou não com os períodos de cheia e seca.

Para o estudo, foram selecionados: 139 imagens MOD13 (índices de vegetação), derivadas do sensor MODIS a bordo da plataforma Terra e disponível no portal EOS Data Gateway (Nasa, 2007a) (Huete et al., 2002), em composição de 16 dias referentes a junho de 2000 a julho de 2006; modelos digitais de elevação, gerados pela missão SRTM (Rabus et al., 2003); e a série histórica de precipitação pluviométrica (milímetros por dia), disponibilizada por TRMM Online Visualization and Analysis System (Nasa, 2007b).

O pré-processamento das imagens MODIS envolveu as etapas de mudança de projeção cartográfica e recorte da área de interesse. Este procedimento foi realizado de forma automática pelo programa MODIS Reprojection Tool (MRT). Neste procedimento, é necessário informar o retângulo referente à área de estudo, a projeção, a resolução espacial e o tipo de interpolação a ser utilizada. Todos os dados foram re-amostrados para $250 \mathrm{~m}$ no sistema de coordenadas geográficas (datum: WGS-84), com intuito de facilitar a integração dos dados em diferentes programas.

Com o objetivo de reduzir a dimensionalidade dos dados, estabeleceu-se um sistema de amostragem com base na altimetria da área de estudo. A escolha da altimetria como parâmetro de amostragem se deveu à relação desta com o regime de inundações. A amostragem teve por base isolinhas geradas automaticamente, com a primeira isolinha localizada na cota de $90 \mathrm{~m}$, e as demais com as eqüidistâncias de $10 \mathrm{~m}$, no total de 11 amostras que se encontram numeradas na Figura 1.

Para obtenção dos valores médios em cada amostra, foi realizado o processo de intersecção entre as curvas de nível e o conjunto de imagens MODIS. Assim, foram extraídos os valores médios, para cada data, do índice enhanced vegetation index (EVI). A escolha deste índice foi feita em razão das características da cobertura vegetal da área de estudo, apresentadas por Abdon et al. (1998) e Pott \& Pott (2004). Esse índice ressalta a estrutura da vegetação e considera a influência da resposta espectral de fundo (Huete et al., 2002); é dado por:

$\mathrm{EVI}=\mathrm{G}(\rho \mathrm{IV}-\rho \mathrm{V}) /(\rho \mathrm{IV}+\mathrm{C} 1 \rho \mathrm{V}-\mathrm{C} 2 \rho \mathrm{A}+\mathrm{L})$ em que: $\mathrm{G}$ é o fator de ganho $(2,5)$; $\rho \mathrm{IV}$ é a reflectância no infravermelho; $\rho \mathrm{V}$ é a reflectância no vermelho; $\mathrm{C} 1$ e C2 são os coeficientes de resistência de aerossóis, dados por 6 e 7,5, respectivamente; $\rho$ A é a reflectância no azul; e L é o ajuste da linha do solo.

A precipitação (mm por dia), estimada pelo TRMM, foi utilizada como dado auxiliar à interpretação dos resultados, uma vez que o pulso de inundação rege a dinâmica do uso e a cobertura do bioma pantaneiro. Esses dados estimados pelo TRMM foram testados por Collischonn et al. (2007), em uma região do Pantanal, que concluíram que essas estimativas estão consistentes e conseguem reproduzir com fidelidade o regime de chuvas. A fim de compatibilizar as análises, os dados de precipitação foram integrados na mesma freqüência de tempo das imagens EVI, de 16 em 16 dias.

A partir dos valores médios de EVI e de precipitação, foi elaborada uma série temporal para cada amostra. Inicialmente, foi realizada a análise exploratória entre estas variáveis, por meio de análise gráfica e de correlação.

Para a análise da dependência temporal dos dados, foi realizado o cálculo da função de autocorrelação para cada uma das 11 amostras. Esta função calcula a correlação de uma série, em diferentes passos no tempo. Ou seja, permite que se entenda melhor a dependência dos dados de uma amostra no tempo. A função de autocorrelação é a representação gráfica do coeficiente de autocorrelação, em função dos diversos "lags" (passos entre os elementos da série) dos dados (Jenkins \& Box, 1976), e em sua forma simplificada é expressa como:

$\mathrm{R}(\mathrm{t}, \mathrm{s})=\mathrm{E}\left[\left(\mathrm{X}_{\mathrm{t}}-\mu\right)\left(\mathrm{X}_{\mathrm{st}}-\mu\right)\right] / \sigma^{2}$

em que: $\mathrm{E}$ é a esperança do produto das variáveis aleatórias; $X_{t}$ é o valor da variável aleatória no tempo t; $X_{\mathrm{s}}$ é o valor da variável aleatória no "lag" s; $\sigma^{2}$ é a variância; e $\mu$ é média da série. $\mathrm{O}$ domínio da função está entre [-1,1], em que valores próximos a um (1) indicam correlação positiva e valores próximos a menos um (-1) indicam a correlação negativa entre os dados.

Com o objetivo de se avaliar a similaridade da resposta espectro-temporal das diferentes amostras no espaço, foi realizada a análise de agrupamentos hierárquicos. Neste método, a distância máxima entre os grupos de amostras é estimada por meio da matriz de distância euclidiana entre os pares de amostras. Para o processo de agrupamento, foi utilizado o método de ligação completa entre os grupos ("farthest neighbor") (Sharma, 1996). Como as amostras estão espacialmente 
distribuídas, esta análise permite inferir sobre a similaridade espectro-temporal no espaço. Espera-se que amostras espacialmente próximas e com cobertura similar tendam a ter comportamento espectro-temporal análogo, o que resulta em distâncias menores entre elas. Os resultados desta análise são apresentados na forma de um dendrograma que permite visualizar a distância entre os grupos no espaço (Sharma, 1996).

Como forma de representar a variabilidade espectrotemporal de cada pixel da área de estudo, foi aplicada a análise de componentes principais às 139 imagens. A técnica de componentes principais permite reduzir a dimensionalidade dos dados e representar a variabilidade no tempo de cada pixel que compõe a imagem (Mather, 1999).

\section{Resultados e Discussão}

As 11 amostras apresentaram comportamento temporal periódico, que coincidiu com a variabilidade da precipitação na área de estudo. Verificou-se que a flutuação temporal do EVI (Figura 2 A) coincidiu com a flutuação temporal da precipitação (Figura 2 B). Esse resultado tem uma importância muito grande para o manejo da região, uma vez que o EVI representa a produtividade primária do sistema (Huete et al., 2002), base da produtividade dos demais segmentos da cadeia alimentar, tanto para a fauna silvestre quanto para a bovinocultura extensiva, principal atividade econômica regional. Pode também auxiliar nos trabalhos de valoração tanto econômica quanto ecológica (Viglizzo \& Frank, 2006). Observou-se que os picos dessas variáveis ocorreram em janeiro, época em que o desenvolvimento vegetativo atinge a plenitude (Goltz et al., 2007).

Com base nessa análise gráfica, realizou-se a análise de correlação entre o EVI e a precipitação, para se verificar se havia retardo na resposta espectral de cada amostra, com relação à precipitação. A maior correlação positiva entre o EVI e a precipitação ocorreu no mesmo período da chuva e variou entre 0,55 e 0,60 (Figura 3). Isso significa que o tempo necessário, para que a vegetação alcançasse o máximo de resposta
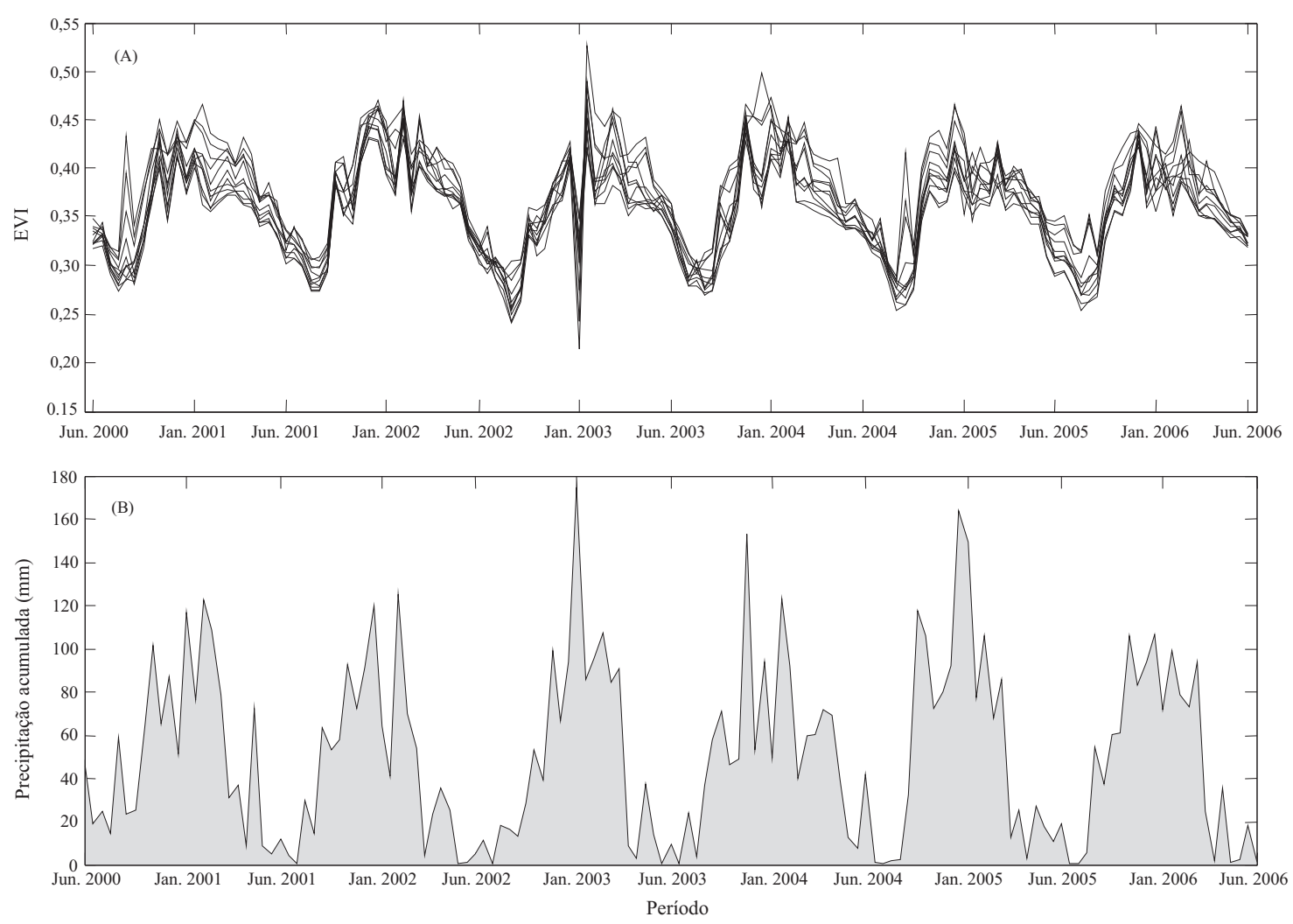

Figura 2. Comportamento temporal das variáveis "enhanced vegetation index" (EVI) e precipitação acumulada, durante o período de junho de 2000 a junho de 2006. 
espectral, coincidiu com o tempo em que a precipitação alcançou o seu máximo. Resultados semelhantes foram apresentados graficamente por Antunes \& Esquerdo (2007) e Goltz et al. (2007). Diferentes fitofisionomias que recebem diferentes quantidades de chuva, em diferentes locais, respondem diferentemente quanto ao retardo na resposta espectral, porém, como a presente análise integra essas variações, a resposta do sistema como um todo apresentou comportamento mais homogêneo, que tendeu ao comportamento monomodal. Entre 48 e 64 dias de defasagem, a correlação foi nula, o que indica que não houve mais efeito da precipitação sobre a resposta espectral. Após 80 dias, a correlação tornou-se inversa, pois, com esta defasagem, ocorreu a máxima diferença entre as fases deste sinal. Ou seja, ocorreram valores baixos de precipitação e altos de índice e vice-versa (Figura 3). Correlações semelhantes foram obtidas por Chaoka et al. (2007), em um estudo que aborda a relação entre o "normalized difference vegetation index" e a precipitação.

O comportamento da correlação entre EVI e precipitação foi análogo em todas as amostras (Figura 3). Com base nessa informação, avaliou-se a correlação entre os valores de EVI para cada amostra no tempo (Figura 4). Os resultados se repetiram, e o comportamento da autocorrelação foi semelhante para todas as amostras (Figura 4 A). Assim, selecionou-se apenas a amostra 6 para exemplo (Figura 4 B). Observou-se na Figura 4 B a existência de um ciclo,

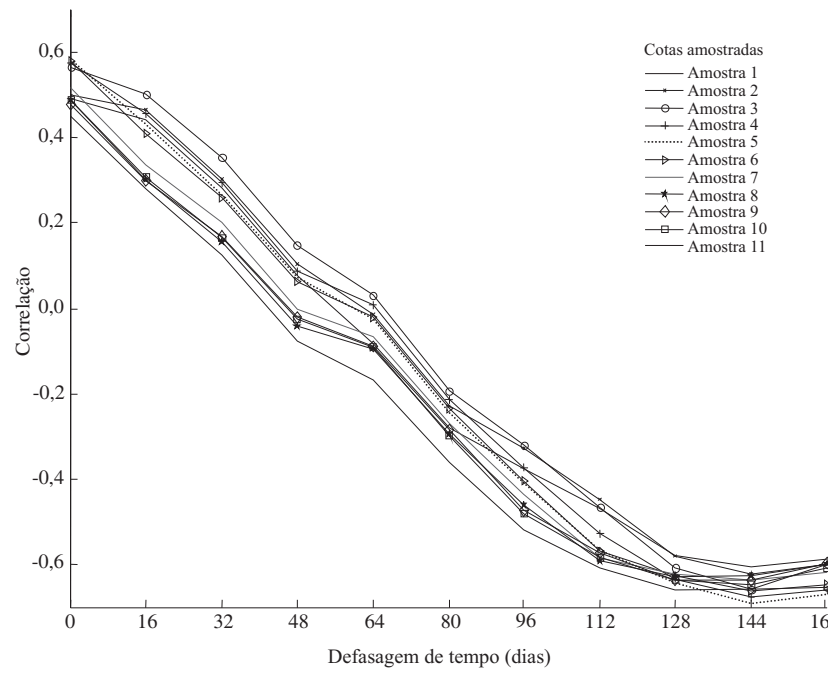

Figura 3. Correlação entre "enhanced vegetation index" e precipitação (mm por dia), com defasagem no tempo, por amostra. relacionado ao próprio período de precipitação. Esse resultado concorda com o obtido da correlação entre o EVI e a precipitação (Figura 3) e com os apresentados por Goltz et al. (2007).

A análise do comportamento temporal das variáveis precipitação e EVI (Figuras 2, 3 e 4) indicou que houve um padrão periódico que concordava com o regime de chuvas. Entretanto, pelo dendrograma, verificou-se que as amostras formaram dois agrupamentos (Figura 5): um com as amostras 1, 2 e 3 , e outro composto pelas demais.
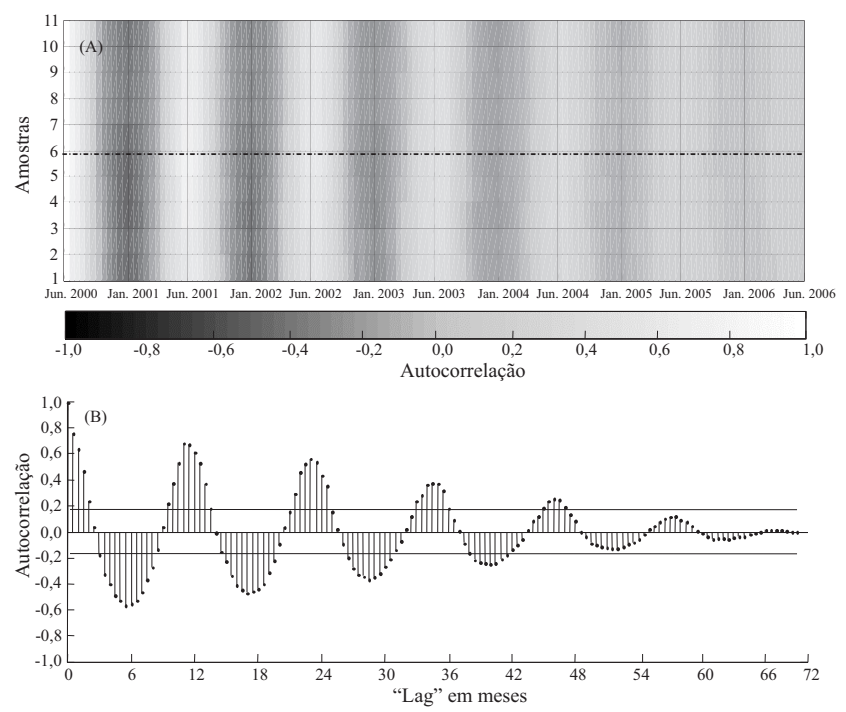

Figura 4. Autocorrelação entre os valores de "enhanced vegetation index" para as 11 amostras (A) e autocorrelação para a amostra $6(\mathrm{~B})$.

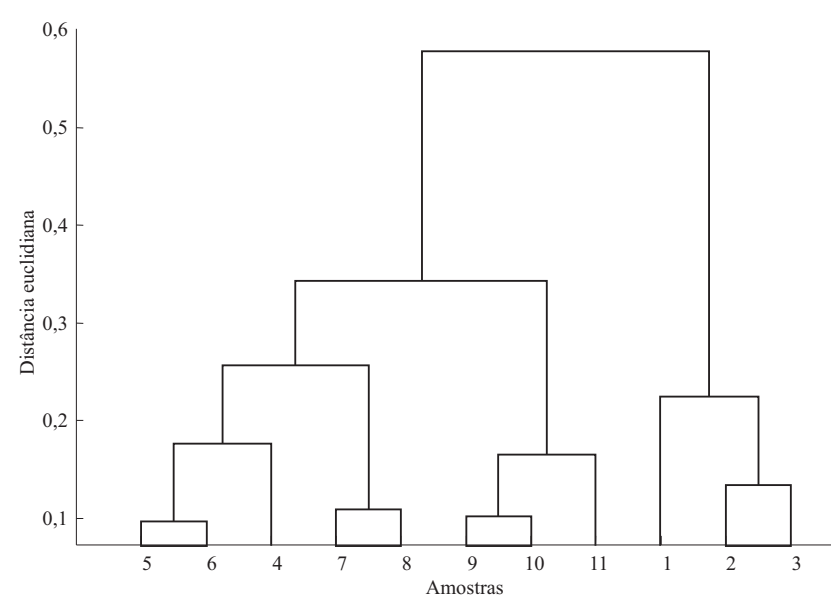

Figura 5. Dendrograma com os agrupamentos entre as cotas amostradas e respectivas distâncias entre os valores médios de "enhanced vegetation index". 
Essa diferenciação entre as amostras 1, 2 e 3 e as demais direcionou o estudo em toda a área, por meio da análise de componentes principais (Figura 6). Nesse caso, a primeira e a segunda componentes principais concentraram $66 \%$ de toda a variância da série de imagens. A partir da terceira componente, os autovalores se tornaram assintóticos, o que indica que nessas componentes houve maior influência de ruídos do que nas duas primeiras componentes. Destacaram-se quatro comportamentos bem definidos. Houve aumento na variabilidade dos valores de EVI, quando se compararam as amostras pontuais com a média de todas as amostras, apresentadas na Figura 2: as áreas em tons escuros, compostas principalmente por áreas permanentemente alagadas, tiveram valores de EVI baixos e com grande variabilidade (Figura 6 A); as áreas de tons claros, compostas por áreas não inundáveis e com vegetação, tiveram valores de EVI que seguem a sazonalidade da vegetação (Figura 6 B); áreas em tons verdes, compostas por áreas inundáveis, mas com maior resposta de vegetação (Figura 6 C); e áreas em tons avermelhados, com vegetação localizada em áreas próximas à rede de drenagem (vegetação ripária), que sofrem pequena influência da sazonalidade da precipitação (Figura 6 D). Resultados semelhantes de área inundáveis e permanentemente inundadas foram obtidos por Padovani et al. (2005), Antunes \& Esquerdo (2007), Goltz et al. (2007) e Lacruz \& Sousa Júnior (2007) com métodos distintos.
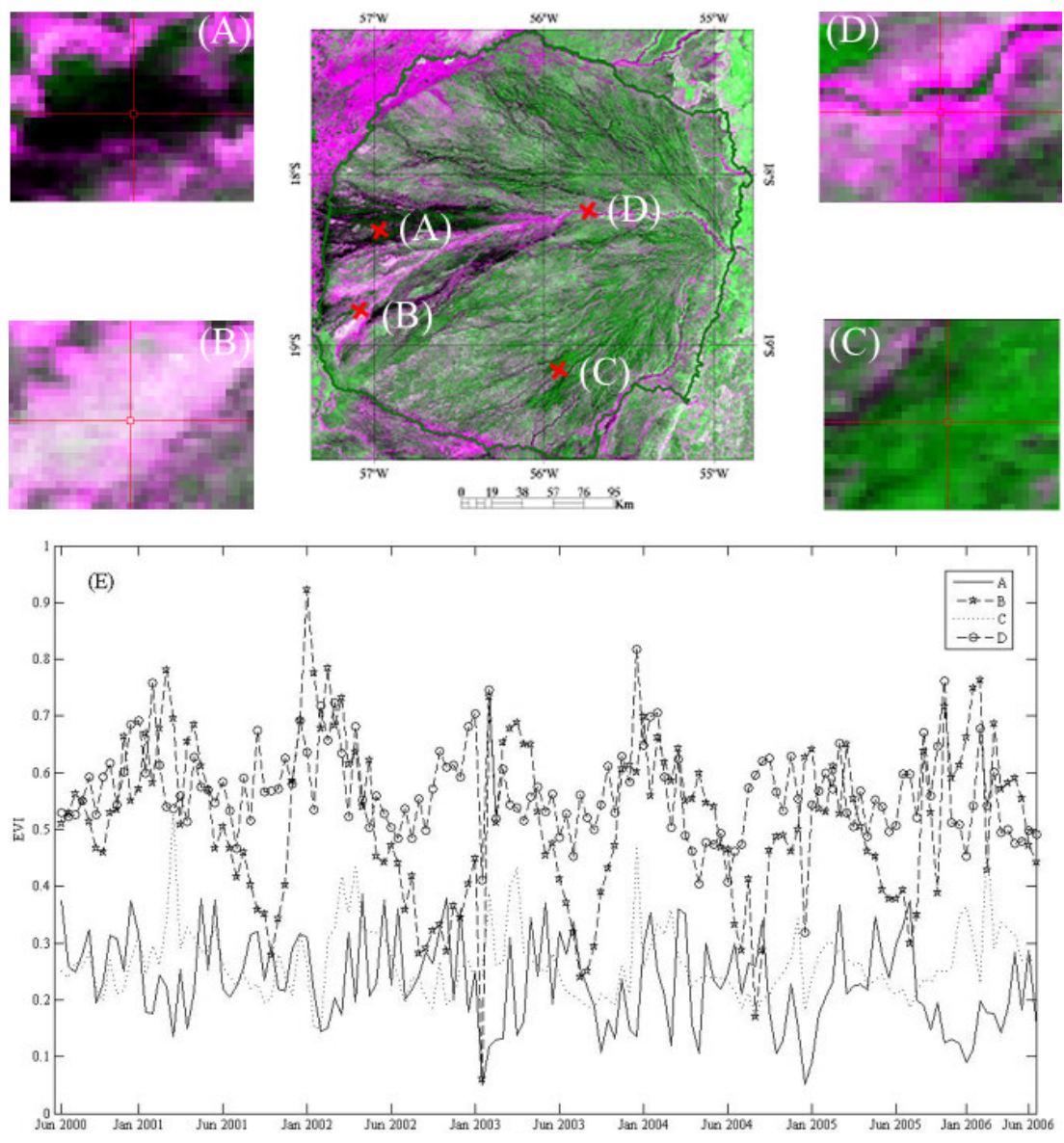

Figura 6. Composição colorida RGB da área de estudo, formada respectivamente pela primeira e segunda componentes e primeira componente principal. A) área com valores baixos na primeira e segunda componentes; B) área com valores altos na primeira e segunda componentes; C) áreas com valores altos na segunda e baixos na primeira componente; D) área com valores altos na primeira e baixos na segunda componente; E) comportamento temporal do EVI nos elementos selecionados. 
A paisagem abrangida pelas amostras 1, 2 e 3 (Figura 1) localiza-se em áreas mais alagadiças, que permanecem nesta condição por um período de tempo maior. Isto ocorre porque o escoamento superficial na região é maior de leste para oeste, do que de norte para sul. Além disso, a área de abrangência dessas amostras apresenta declividade menor do que o restante da área de estudo; conseqüentemente, a área permanece mais tempo inundada do que a área das outras amostras. Ademais, a região das amostras 1, 2 e 3 recebe grande influência das inundações do rio Paraguai, o que a mantém inundada por mais tempo. Este resultado concorda com o do agrupamento observado no dendrograma (Figura 5), que diferenciou essas amostras das demais. Assim, este método permite melhor compreensão da dinâmica espaço-temporal e pode ser aplicado como ferramenta auxiliar no manejo da flora e fauna silvestres, bem como na bovinocultura da região.

\section{Conclusões}

1. A resposta espectral das amostras tem padrão periódico e é influenciada pela precipitação.

2. Há relação entre a posição geográfica e a resposta espectro-temporal.

3. É possível distinguir as áreas permanentemente alagadas, inundáveis e secas.

4. O método apresentado pode ser utilizado para monitorar o bioma pantaneiro.

\section{Agradecimentos}

Ao Conselho Nacional de Desenvolvimento Científico e Tecnológico e à Coordenação de Aperfeiçoamento de Pessoal de Nível Superior, pelo financiamento parcial deste trabalho.

\section{Referências}

ABDON, M. de M.; SILVA, J. dos S.V. da; POTT, V.J.; POTT, A.; SILVA, M.P. da. Utilização de dados analógicos do Landsat-TM na discriminação da vegetação de parte da sub-região da Nhecolândia no Pantanal. Pesquisa Agropecuária Brasileira, v.33, p.1799-1813, 1998.

ADAMOLI,J.Zoneamento ecológico do Pantanal baseadonoregime de inundações. In: ENCONTRO SOBRE SENSORIAMENTO REMOTO APLICADO A ESTUDOS NO PANTANAL, 1., 1995, Corumbá. Anais. São José dos Campos: Inpe, 2005. p.15-17.

ANTUNES, J.F.G.; ESQUERDO, J.C.D.M. Geração automática de produtos derivados de imagens AVHRR-NOAA para monitoramento de áreas inundáveis do Pantanal. Revista Brasileira de Cartografia, v.59, p.115-122, 2007.

CHAOKA, R.T.; ALEMAW, B.F.; TSIGE, D.M. Modeling and understanding the relationship between vegetation and rainfall of a tropical watershed using remote sensing data and GIS. Journal of Spatial Hydrology, v.7, p.47-61, 2007.

COLLISCHONN, B.; ALLASIA, D.; COLLISCHONN, W.; TUCCI, C.E.M. Desempenho do satélite TRMM na estimativa de precipitação sobre a bacia do Paraguai superior. Revista Brasileira de Cartografia, v.59, p.93-99, 2007.

EMBRAPA. Sistema brasileiro de classificação de solos. Rio de Janeiro: Embrapa Solos, 2006. 306p.

GOLTZ, E.; BRANDÃO, D.; TOMÁS, L.; MANTELLI, L.R.; ADAMI, M.; SHIMABUKURO, Y.E.; FORMAGGIO, A.R. Utilização de índices espectrais de vegetação do sensor MODIS na determinação de áreas suscetíveis a alagamento no Pantanal sulmatogrossense. Revista Brasileira de Cartografia, v.59, p.35-44, 2007.

HUETE, A.; DIDAN, K.; MIUIRA, T.; RODRIGUEZ, E.P.; GAO, X.; FERREIRA, L.G. Overview of the radiometric and biophysical performance of the MODIS vegetation indices. Remote Sensing of Environment, v.83, p.195-213, 2002.

IBGE. Instituto Brasileiro de Geografia e Estatística. Manual técnico da vegetação brasileira. Rio de Janeiro: IBGE, 1992. 92p.

JENKINS, G.M.; BOX, G.E.P. Time series analysis: forecasting and control. $2^{\text {nd }}$ ed. Oakland: Holden-Day, 1976. 575p.

JUSTICE, C.O.; TOWNSHEND, J.R.G.; VERMOTE, E.F.; MASUOKA, E.; WOLFE, R.E.; SALEOUS, N.; ROY, D.P.; MORISETTE, J.T. An overview of MODIS land data processing and product status. Remote Sensing of Environment, v.83, p.3-15, 2002.

LACRUZ, M.S.P.; SOUSA JÚNIOR, M. de A. Uso de séries temporais EVI/MODIS e análise harmônica para o estudo da bacia do rio Taquari. Revista Brasileira de Cartografia, v.59, p.9-15, 2007.

MATHER, P.M. Computer processing of remotely-sensed images: an introduction. $2^{\text {nd }}$ ed. Chichester: John Wiley \& Sons, 1999. 292p.

NASA. National Aeronautics and Space Administration. Earth observing system data gateway. Disponível em: <http:// edcimswww.cr.usgs.gov/pub/imswelcome/>. Acesso em: 15 out. 2007a.

NASA. National Aeronautics and Space Administration. Monthly TRMM and other data sources rainfall estimate. Disponível em: $<$ http://disc2.nascom.nasa.gov/Giovanni/tovas/TRMM_V6.3B43. shtml>. Acesso em: 15 out. 2007b.

PADOVANI, C.R.; ASSINE, M.L.; VIERA, L.M. Inundação no leque aluvial do rio Taquari. In: GALDINO, S.; VIEIRA, L.M.; PELLEGRIN, L.A. (Ed.). Impactos ambientais e socioeconômicos na bacia do rio Taquari: Pantanal. Corumbá: Embrapa Pantanal, 2005. p.183-198.

POTT, A.; POTT, V.J. Features and conservation of the Brazilian Pantanal wetland. Wetlands Ecology and Management, v.12, p.547-552, 2004. 
RABUS, B.; EINEDER, M.; ROTH, A.; BAMLER, R. The shuttle radar topography mission- a new class of digital elevation models acquired by spaceborne radar. ISPRS Journal of Photogrammetry Engineering and Remote Sensing, v.57, p.241-262. 2003.

SHARMA, S. Applied multivariate techniques. New York: John Wiley \& Sons, 1996. 512p.

SHIMABUKURO, Y.E.; NOVO, E.M.; PONZONI, F.J. Índice de vegetação e modelo linear de mistura espectral no monitoramento da região do Pantanal. Pesquisa Agropecuária Brasileira, v.33, p.1729-1737, 1998.
SILVA, J. dos S.V. da; ABDON, M. de M. Delimitação do Pantanal brasileiro e suas sub-regiões. Pesquisa Agropecuária Brasileira, v.33, p.1703-1711, 1998.

VIGLIZZO, E.F.; FRANK, F.C. Land-use options for Del Plata Basin in South America: tradeoffs analysis based on ecosystem service provision. Ecological Economics, v.57, p.140-151, 2006.

WOLFE, R.E.; NISHIHAMA, M.; FLEIG, A.J.; KUYPER, J.A.; ROY, D.P.; STOREY, J.C.; PATT, F.S. Achieving sub-pixel geolocation accuracy in support of MODIS land science. Remote Sensing of Environment, v.83, p.31-49, 2002.

Recebido em 30 de abril de 2008 e aprovado em 9 de setembro de 2008 